\title{
The Emerging Role of Drug-Induced Sleep Endoscopy in the Management of Obstructive Sleep Apnea
}

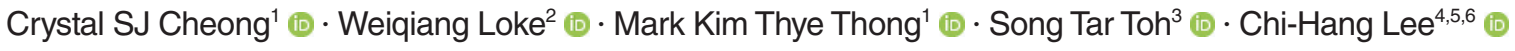 \\ ${ }^{1}$ Department of Otolaryngology-Head and Neck Surgery, National University Hospital, Singapore; ${ }^{2}$ Faculty of Dentistry, National University of \\ Singapore, Singapore; ${ }^{3}$ Department of Otolaryngology, Singapore General Hospital, SingHealth Duke-NUS Sleep Centre, Singapore; \\ ${ }^{4}$ Department of Cardiology, National University Heart Centre Singapore, Singapore, ${ }^{5}$ Department of Medicine, Yong Loo Lin School of Medicine, \\ National University of Singapore, Singapore; ${ }^{6}$ Cardiovascular Research Institute, National University of Singapore, Singapore
}

Obstructive sleep apnea is a prevalent sleep disorder characterized by partial or complete obstruction of the upper airway. Continuous positive airway pressure is the first-line therapy for most patients, but adherence is often poor. Alternative treatment options such as mandibular advancement devices, positional therapy, and surgical interventions including upper airway stimulation target different levels and patterns of obstruction with varying degrees of success. Drug-induced sleep endoscopy enables the visualization of upper airway obstruction under conditions mimicking sleep. In the era of precision medicine, this additional information may facilitate better decision-making when prescribing alternative treatment modalities, with the hope of achieving better adherence and/or success rates. This review discusses the current knowledge and evidence on the role of drug-induced sleep endoscopy in the non-positive airway pressure management of obstructive sleep apnea.

Keywords. Surgery; Mandibular Advancement Device; Obstructive Sleep Apnea; Endoscopy

\section{INTRODUCTION}

Obstructive sleep apnea (OSA) affects nearly one billion people worldwide based on statistics published in 2019 , with a prevalence exceeding $50 \%$ in some countries [1]. While snoring and excessive daytime sleepiness are the commonest and most emphatic complaints of patients, it is the resultant cardiovascular sequelae that have the direst consequences on health [2-4]. Continuous positive airway pressure (CPAP) has long been commonly accepted as the first-line treatment for OSA. However, it is well-known for having low levels of acceptance and poor adherence [5]. Alternative treatments such as mandibular advancement devices (MADs), positional therapy, and upper airway surgery

\footnotetext{
- Received August 11, 2020

Revised September 28, 2020

Accepted September 29, 2020

- Corresponding author: Crystal SJ Cheong

Department of Otolaryngology-Head and Neck Surgery, National

University Hospital, 1E Kent Ridge Rd, NUHS Tower Block Level 7,

Singapore 119228, Singapore

Tel: +65-6772-5370, Fax: +65-6775-3820

E-mail: crystal_sj_cheong@nuhs.edu.sg
}

(including upper airway stimulation) may be considered for patients who cannot tolerate or accept CPAP [6-8].

OSA therapy is traditionally guided by the results of a sleep study that determines the severity of OSA by calculating the average number of respiratory events per hour of sleep, otherwise known as the apnea-hypopnea index (AHI). The AHI, however, does not reflect the complicated pathophysiology behind OSA; it merely reflects the resultant respiratory compromise. Drilling down to the contributory roles of various anatomical and/or non-anatomical pathophysiological mechanisms behind OSA in each patient is useful in order to better select appropriate treatment methods [9]. Various algorithms utilizing metrics apart from the AHI that are reported during routine clinical polysomnography (PSG) have been created to do this [10-12], but they are still not widely employed in the clinical setting. Analysis of inspiratory flow shape during PSG may help to pinpoint the specific site of obstruction, particularly in cases with an isolated collapsing pharyngeal structure [13], but obstruction often occurs at multiple levels in OSA $[14,15]$. Sleep studies therefore play a limited role in determining the suitability of alternative treatments at the individual level.

Copyright (C) 2021 by Korean Society of Otorhinolaryngology-Head and Neck Surgery.

This is an open-access article distributed under the terms of the Creative Commons Attribution Non-Commercial License (https://creativecommons.org/licenses/by-nc/4.0) which permits unrestricted non-commercial use, distribution, and reproduction in any medium, provided the original work is properly cited. 
Table 1. Summary of potential roles of DISE in determining treatment options for OSA

Alternative treatment for OSA
$\begin{aligned} & \text { Mandibular advancement device } \\ & \text { Retrolingual collapse during DISE and expansion of the retrolingual space with mandibular advancement are } \\ & \text { predictive of treatment success. } \\ & \text { A simulation bite may mimic the actual effects of a mandibular advancement device more accurately than a jaw thrust. } \\ & \text { Improvement in collapse when the patient is in the lateral (compared to supine) position is predictive of success. } \\ & \text { Obstruction at the level of the tongue base and epiglottis will change most significantly with position. } \\ & \text { To select an appropriate surgical technique for the site of obstruction. } \\ & \text { DISE findings may alter the initial surgical plan. } \\ & \text { Retrolingual obstruction may suggest lower success rates with uvulopalatopharyngoplasty alone. } \\ & \text { DISE findings may not be predictive of success for certain soft tissue surgical procedures (e.g., tongue base surgery) } \\ & \text { and may not improve the overall surgical success rate. } \\ & \text { Oropharyngeal wall obstruction may suggest a higher success rate with maxillomandibular advancement than soft } \\ & \text { tissue surgery. } \\ & \text { Pediatric patients at high risk of persistent OSA may benefit from DISE even before undergoing tonsillectomy and } \\ & \text { adenoidectomy. } \\ & \text { To determine hypoglossal nerve stimulator candidacy, as complete concentric collapse of the palate has been } \\ & \text { associated with poorer outcomes. }\end{aligned}$
Upper airway stimulation

DISE, drug-induced sleep endoscopy; OSA, obstructive sleep apnea.

Drug-induced sleep endoscopy (DISE) has emerged as a safe and useful technique that allows direct visualization of the anatomy and collapsibility of the upper airway via nasoendoscopy while the patient is sedated [16-18]. It is a relatively simple procedure, and complications such as central apnea and deep desaturation requiring intubation are infrequent [19]. Since it was first described in 1991 [20], anecdotal reports, case series, and prospective registries on DISE have been published. Propofol, midazolam, and dexmedetomidine are the most commonly employed anesthetic drugs. Administration techniques vary across centers, ranging from bolus doses to regular injection doses and continuous infusions. Clinical assessments of unconsciousness with vocal and/or tactile stimulation, as well as indices such as the bispectral index score, help determine the appropriate level of sedation. The variation in techniques worldwide reflects limitations in the realm of DISE; specifically, no drug currently achieves or mimics natural sleep perfectly, and there is no established "best" way at present to perform DISE despite many recommendations. Multiple classification systems have been de-

\section{H I G G H L I I G H H T S}

- Drug-induced sleep endoscopy (DISE) allows visualization of upper airway collapsibility for non-continuous positive airway pressure therapy of obstructive sleep apnea (OSA).

- Jaw thrust or simulation bite predicts benefits from a mandibular advancement device.

- Positional maneuvers during DISE assess the potential utility of positional therapy.

- Knowledge of collapse levels and configurations enables customization of the surgical plan.

- More studies are required to demonstrate the efficacy of DISE in the management of OSA. vised over the years to report endoscopic findings accurately and succinctly, but none has been universally adopted [21,22]. As CPAP functions by splinting the entire length of the upper airway open, patients on CPAP therapy do not usually undergo DISE to determine the exact sites of obstruction, but may benefit from a more precise evaluation if it is necessary to troubleshoot the mechanism of CPAP failure [23]. This review provides an overview of the current knowledge and evidence on the role of DISE in non-CPAP management alternatives for OSA. Many studies in the current literature are retrospective, non-randomized, and heterogeneous, making it challenging to conduct headto-head comparisons or wide-scale validation of specific techniques. We discuss some unique studies that reveal different perspectives in this field, and summarize what DISE can offer in the management of OSA (Table 1).

\section{ROLE OF DISE IN PRESCRIBING MADs}

The MAD is an oral appliance recommended for adult OSA patients who are intolerant of CPAP or prefer an MAD [24]. By mechanically advancing the mandible, MADs enlarge the upper airway laterally, predominantly at the velopharynx, due to stretching of the soft tissue connecting the mandibular rami, tongue, soft palate and lateral walls $[25,26]$. The tongue muscles are shifted forward [25,27], and the airway becomes less collapsible. It is effective in reducing breathing disturbances, AHI, and excessive daytime sleepiness. Compared to CPAP, MADs achieve similar health-related outcomes, but have better acceptance and tolerance rates [28].

Being able to accurately predict whether an MAD will be beneficial is advantageous, as device and follow-up costs can be sizable. To simulate wearing an MAD, the mandible is manually advanced by $5-10 \mathrm{~mm}$ during DISE. If the upper airway caliber 

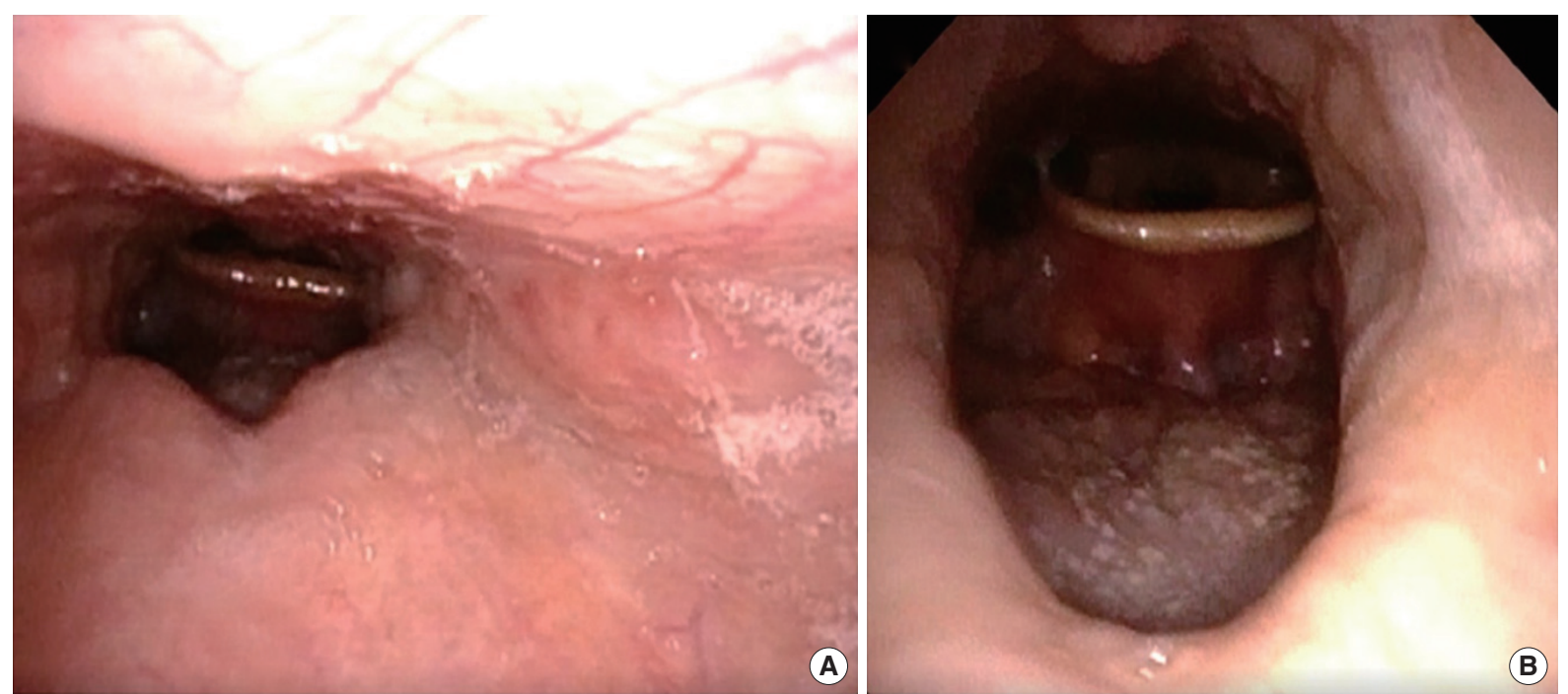

Fig. 1. The narrow retropalatal and retrolingual spaces seen during drug-induced sleep endoscopy (A) expand when a jaw thrust maneuver is performed (B).

increases significantly in response to manual advancement of the mandible, it can be predicted that MAD therapy will be helpful (Fig. 1).

One of the earliest accounts of DISE being able to predict successful MAD therapy was published in 2005 [29]. The study described 19 patients who underwent MAD therapy after diagnostic DISE revealed multilevel obstruction $(n=18)$ or isolated tongue base obstruction $(n=1)$. Gentle mandibular advancement was performed in 17 of these patients, improving airway patency and snoring in all. Follow-up DISE with an MAD after 8-25 months showed the following: three patients had a clear airway without snoring, eight had marked subjective improvements in both the airway and snoring, five improved but had residual palatal snoring, and one did not demonstrate any change. The patient with no change subsequently experienced symptom resolution after further advancement of his MAD, indicating that it had not been titrated adequately. PSG done at least 2 months after subjectively successful MAD use showed an overall decrease in the median AHI from 28 (range, 14-62) to 6 (range, 0.3-17) events/hr. Treatment success (defined as AHI $<10$ events/hr) was achieved in $74 \%$ of the patients. The authors suggested that DISE could serve as a diagnostic test to identify patients with obstruction configurations that would benefit from an MAD, and as a prognostic indicator for MAD therapy.

More recently, a non-randomized retrospective case control study compared the MAD treatment outcomes of 20 OSA patients who had undergone DISE before using an MAD (DISE group) versus 20 who had not (non-DISE group) [30]. The DISE and non-DISE cohorts were matched for age, body mass index, and pre-MAD PSG characteristics. The DISE group was selected for MAD therapy because of the observation that the jaw thrust maneuver during DISE led to significant improvement in the retrolingual and retropalatal airspace. The DISE group was found to have a higher rate of treatment success, defined as a post-treatment AHI $<20$ events $/ \mathrm{hr}$ with a $50 \%$ improvement $(75 \%$ of DISE patients vs. $50 \%$ of non-DISE patients; $P=0.09$ ), and a larger proportion dropped to $<5$ events $/ \mathrm{hr}$ ( $45 \%$ of DISE patients vs. $15 \%$ of non-DISE patients; $P=0.04$ ). There was a significantly greater decrease in the AHI in the DISE group than in the non-DISE group ( $31.54 \pm 23.19$ to $7.93 \pm 6.03$ events/hr vs. $29.81 \pm 19.36$ to $14.67 \pm 12.23$ events/hr, respectively; $P=0.04$ ). Although limitations include small sample size, lack of randomization, and varying levels of sleep studies used in post-treatment evaluation, this study suggests that enlargement of the retrolingual and retropalatal airway during a jaw thrust is associated with effective MAD treatment.

To determine whether manually pulling the mandible forward or using a simulation bite was more accurate at predicting MAD outcomes, a center performed DISE on 200 patients with sleepdisordered breathing who were considering MAD treatment [31]. Multilevel obstruction was predominantly seen $(87.2 \%)$, with combined palatal and tongue base collapse $(34.4 \%)$ being the most common pattern. The upper airway was assessed first with a simulation bite in situ. The simulation bite was then removed to return the patient to baseline, and finally the patient was evaluated using a chin-lift maneuver to achieve maximal protrusion of the mandible. After review by a dental sleep professional who was blinded to the DISE findings, 110 OSA patients (of whom $53.6 \%$ had mild OSA) eventually completed MAD titration and a repeat PSG with MAD in situ. Unfortunately, this study did not have a control group. Positive treatment response, defined as a reduction in AHI of $\geq 50 \%$, was achieved in $69 \%$ of patients. After adjusting for sex, age, body mass index, AHI, and positional dependency, a complete absence of collapse while wearing the 
simulation bite during DISE was independently associated with a positive treatment response $(P=0.007)$. The presence of palatal collapse $(P=0.02)$ and absence of hypopharyngeal collapse $(P=$ 0.03 ) during baseline DISE were also independent predictors of positive treatment response. The use of a simulation bite during DISE to predict the treatment outcome showed a sensitivity of $91 \%$, a specificity of $53 \%$, a positive likelihood ratio of 1.96 , and a negative likelihood ratio of 0.16 . Interestingly, the chin-lift maneuver was not found to have a statistically significant association with upper airway caliber or treatment response. The authors postulated that the simulation bite was a significant predictor because it added a certain amount of vertical opening, which also occurs with an MAD, but not with a chin-tilt or jaw thrust. Another study reported only slight to moderate agreement in the degree of obstruction and configuration of the upper airway between a jaw thrust and a boil-and-bite MAD during DISE [32]. Some have pointed out that the discomfort from a jaw thrust can awaken or reduce the depth of sedation of the patient, which may play a part in improving the degree of obstruction [33,34].

A recent study investigated the use of collapse patterns seen during DISE as a means of prognosticating MAD success and failure [35].This study analyzed 72 patients who were prescribed an MAD, completed baseline DISE, and underwent a 3-month follow-up PSG. The presence of tongue base collapse during DISE was associated with a 3.69 times higher odds ratio $(P=0.013)$ for achieving response, defined as a decrease in AHI of $\geq 50 \%$. Complete concentric collapse at the palate (CCCp) and complete laterolateral oropharyngeal collapse resulted in $5.32(P=0.234)$ and $6.62(P=0.033)$ times higher odds of worsening baseline AHI (treatment deterioration) respectively, after adjusting for AHI and body mass index. In a retrospective analysis, the authors noted that using tongue base collapse and CCCp configu- rations to advise for and against MAD prescription in their subgroup of moderate to severe OSA patients would have increased the proportion of patients who responded and reduced the proportion of patients who deteriorated by approximately $50 \%$, respectively.

Many of the published studies on DISE and MAD are retrospective. Selection bias is also a major issue as those recruited for MAD tended to have less severe OSA, and patients deemed not likely to benefit were not recruited for MAD use in the first place. Nonetheless, based on the currently available information, it appears that most patients who have improved airway dimensions with mandibular advancement during DISE will benefit from an MAD. Application of a custom-made simulation bite in preadjusted maximum comfortable protrusion when clinically feasible may improve predictive accuracy.

\section{ROLE OF DISE IN PRESCRIBING POSITIONAL THERAPY}

A large proportion of OSA patients have positional OSA because gravitational forces worsen upper airway collapse when they are supine [36-38]. Positional therapy works by preventing supine sleep; techniques include special pillows and binders, positional alarms, vests, and the older method of sewing tennis balls into a pocket on the back of a shirt. Although equally effective at reducing respiratory indices in patients with mild OSA, the more cost-effective tennis ball technique has lower adherence and poorer quality of life outcomes than sleep position trainers [39].

Turning the patient to the lateral position during DISE can simulate the possible effect of positional therapy. It has been suggested that turning both the head and trunk lateral is more
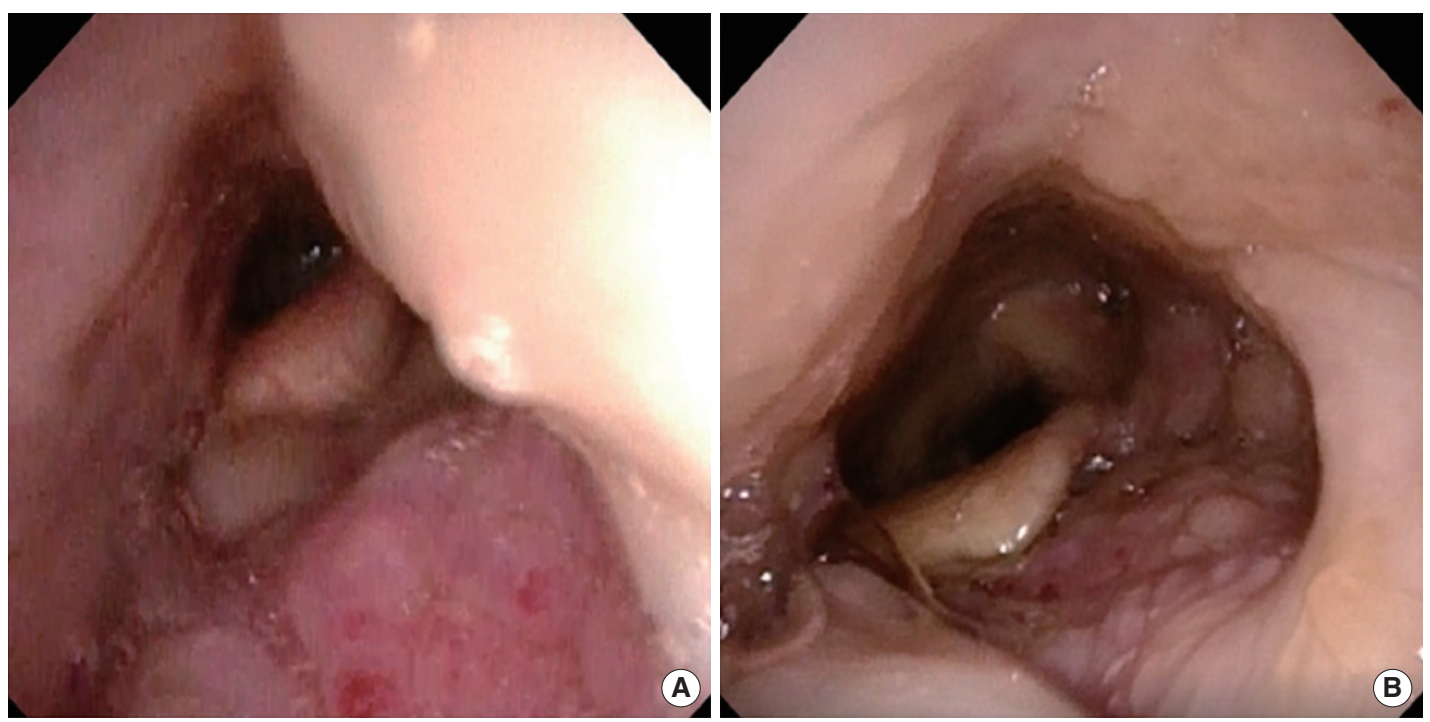

Fig. 2. The tongue base collapse seen in supine position (A) during drug-induced sleep endoscopy improves with head turning (B). The effect of palatal coupling is also visible in (B). 
representative of non-supine sleep positioning in patients with positional OSA than turning the head alone [40]. Improvement in collapse is highly marked in positional OSA patients, with up to $91 \%$ of positional OSA patients demonstrating at least partial improvement when lateral instead of supine $[41,42]$, suggesting that DISE frequently confirms the positional findings on PSG. DISE may be useful in situations where a patient is certain that his/her non-supine sleep is subjectively better than when supine, but PSG reports inadequate non-supine sleep time. Tongue base and epiglottic collapse improve most with positional change (Fig. 2) $[42,43]$.

A randomized controlled study conducted on patients with residual positional OSA despite MAD treatment showed that the additional use of a sleep position trainer with an MAD resulted in higher therapeutic efficacy, as proven on PSG, than using either of the treatment modalities alone [44]. Positional maneuvers during DISE can assess the feasibility of combination therapy (e.g., MAD or limited surgery with positional therapy) for multilevel collapse, potentially reducing the number of invasive interventions required [40].

\section{ROLE OF DISE IN PLANNING SURGICAL INTERVENTION}

OSA surgery aims to improve upper airway patency by removing structures that cause obstructions, stiffening collapsible areas, and expanding the luminal dimensions [45]. Surgical interventions carry inherent risks, so it is critical that the procedures and patients are appropriately selected to maximize the success rate. Although an awake endoscopic examination is informative and easily performed by otolaryngologists, DISE confers additional information about collapse configurations under sedation. Some surgeons supplement the static clinical examination with the Müller maneuver, but this maneuver is effort-dependent and has been criticized for flaws such as inaccuracy at predicting retrolingual collapse during sedation or sleep (Figs. 3 and 4) [46,47].

Identifying the main contributor(s) of collapse guides decision- making. Lateral pharyngeal wall collapse, for instance, is notoriously difficult to address with soft tissue surgery. Maxillomandibular advancement advances the bony framework of the upper and lower jaws, is highly successful at reducing upper airway collapsibility (especially at the lateral walls), and produces excellent outcomes $[48,49]$. Although it is arguably more major surgery, it is justifiable to propose maxillomandibular advancement surgery as the primary procedure in cases where DISE reveals significant lateral pharyngeal collapse.

Several studies have also investigated the utility of DISE in predicting the likelihood of success following specific surgical procedures. One study found that different patterns of airway obstruction seen on DISE predicted different outcomes after tonsillectomy and uvulopalatopharyngoplasty [50]. Twenty OSA patients with soft palate obstruction identified during the Müller maneuver underwent DISE before surgery. The levels of obstruction seen during DISE were categorized into upper airway ob-

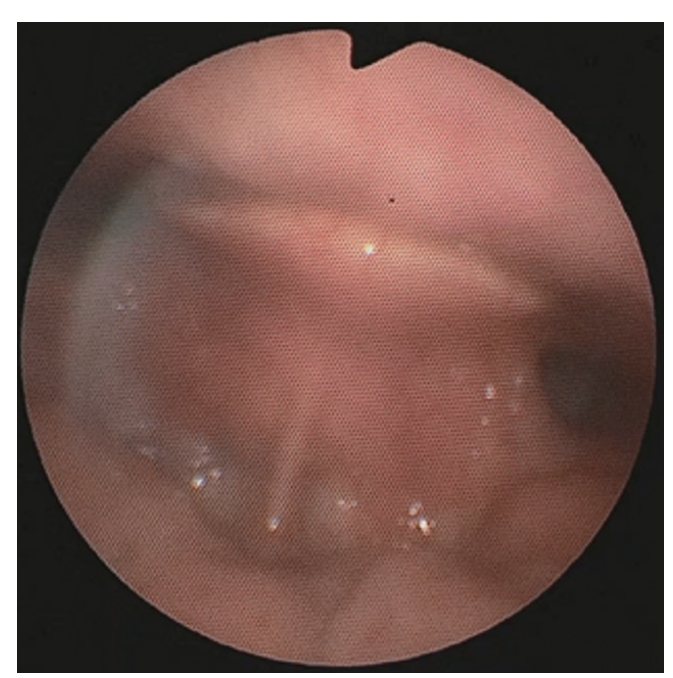

Fig. 4. Rhythmic complete anteroposterior collapse of the epiglottis was seen during drug-induced sleep endoscopy. This did not occur during the clinical examination while the patient was awake, even during the Müller maneuver.
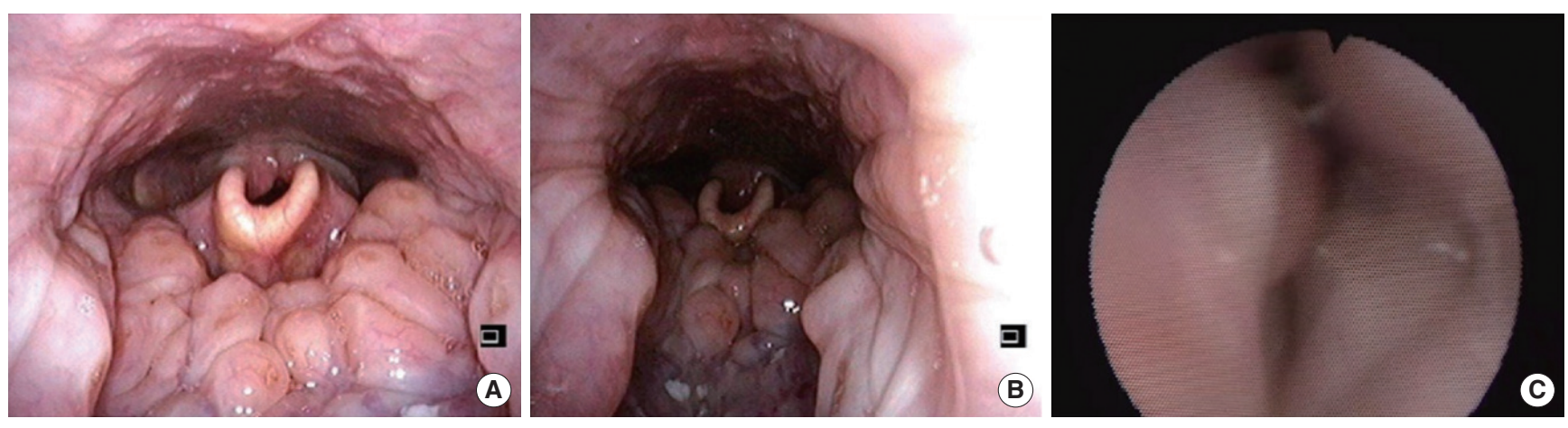

Fig. 3. (A) Endoscopic view of the upper airway at rest. (B) The lateral oropharyngeal walls collapsed partially during the Müller maneuver. (C) However, complete collapse was seen during drug-induced sleep endoscopy. 
struction (i.e., originating from the uvula, soft palate, and/or tonsils) and lower airway obstruction (i.e., originating from the tongue base and/or epiglottis). Surgical success was defined as a decrease in the respiratory disturbance index to $<5$ events/hr or by $\geq 50 \%$. A higher surgical success rate was reported in the group with upper airway obstruction during DISE $(P<0.05)$. All 14 successful cases displayed upper airway obstruction. The group with lower airway obstruction had a lower success rate $(P<0.01)$.

However, the outcomes of other surgical procedures, such as tongue base surgery, are not as predictable. A retrospective case series of 101 patients who underwent transoral robotic surgery found that preoperative DISE findings were not predictive of success or cure, although patients without oropharyngeal lateral wall collapse were more likely to demonstrate improvement [51]. Similarly, a recently published single-center retrospective study reported the surgical outcomes of 95 patients who had undergone tongue base surgery as part of multilevel surgery [52]. No significant difference was found between the group that only underwent a preoperative awake endoscopic examination with the Müller maneuver and the group that underwent DISE in addition to the Müller maneuver, both in terms of percentage of AHI improvement $(47.0 \% \pm 32.0 \%$ vs. $48.3 \% \pm 35.4 \%$, respectively, $P=0.852)$ and surgical success $(42.6 \%$ vs. $45.8 \%$, respectively, $P=0.748$ ), defined as a postoperative $\mathrm{AHI}<20$ events $/ \mathrm{hr}$ with $\geq 50 \%$ improvement in $\mathrm{AHI}$.

A recent multicenter retrospective study involving 275 patients (59\% had severe OSA) highlighted the utility of DISE in prognosticating pharyngeal surgery outcomes in general [53]. All patients underwent preoperative DISE. The distribution of primary structure collapse was as follows: velum, $35 \%$; oropharyngeal lateral walls, $24 \%$; tongue, $39 \%$; and epiglottis, $2 \%$. The overwhelming majority (93\%) underwent palate surgery, and $60 \%$ underwent tongue surgery. Overall, $41 \%$ achieved a surgical response (defined as an AHI decrease of $\geq 50 \%$ and $<15$ events/hr), and the AHI improved from $41 \pm 24$ to $21 \pm 20$ events/hr $(P<0.001)$. A greater AHI decrease was seen for complete than partial velum-related obstruction in patients who underwent palate and tongue resection procedures, but there was no difference in the postoperative AHI $(22.0 \pm 17.4$ vs. $18.6 \pm 17.0$ events/hr, $P=0.44)$. In adjusted analyses, the surgical success rate was approximately $50 \%$ lower for any oropharyngeal lateral wall-related obstruction among all patients, in those with moderate to severe OSA, and in those who underwent isolated palate surgery. Complete tonguerelated obstruction was also associated with a lower surgical success rate in patients with moderate to severe OSA.This study found that velum and epiglottis-related obstructions were not clearly associated with surgical outcomes. The differing conclusion found for velum-related obstructions in this paper may be attributed to tonsillar hypertrophy being an exclusion criterion.

Other issues when considering the potential usefulness of DISE include whether the surgical plan is altered based on information gathered from DISE, and whether such alterations in- crease success rates. Several studies have shown that the surgical plan can be changed in up to $64 \%$ of cases after DISE is performed [54-58].

In the pediatric population, DISE is traditionally performed if adenotonsillectomy, the classical first-line surgical treatment, is ineffective [59]. Patients at high risk of persistent OSA after adenotonsillectomy (e.g., those with small tonsils and adenoids, obesity, age $>7$ years, severe OSA, African-American ethnicity, Down syndrome, craniofacial anomalies, and neuromuscular disorders) may benefit from DISE even before initial surgery [60], as one study showed that $58 \%$ of patients ended up undergoing surgery other than adenotonsillectomy [61]. A DISE-directed intervention at the time of initial surgery in children has been shown to produce symptomatic and objective improvement on repeat PSG [61,62]. Occult or late-onset laryngomalacia, a condition where the arytenoids prolapse into the laryngeal inlet only during sleep, has been reported in children older than those who experience infantile laryngomalacia. This finding would only be obvious during DISE, since it only occurs during sleep. A study of 22 pediatric patients who underwent supraglottoplasty for this finding demonstrated an AHI reduction from 15.4 to 5.4 events/hr $(P<0.001)$, with comparable reductions in AHI for those who had undergone supraglottoplasty alone or in combination with other interventions [63]. Several other studies have also reported successful clinical outcomes of supraglottoplasty, but did not obtain postoperative PSG due to the dramatic clinical improvement noted $[64,65]$.

There are conflicting conclusions regarding the impact of DISE on surgical outcomes. Some studies have suggested that DISE improves success rates, possibly because it aids selection of an appropriate operative technique. A study of 136 patients who underwent uvulopalatopharyngoplasty after demonstrating at least retropalatal obstruction on DISE reported surgical outcomes that were better than historical data [66]. A single-center retrospective analysis of 87 patients found that multiple procedures for multilevel obstruction were performed less frequently in those who underwent DISE than in those who did not ( $8 \%$ vs. $60 \%$, $P<0.001)$ [67]. Nonetheless, the mean postoperative AHI was lower in the DISE group than in the non-DISE group (10 vs. 19 events/hr, $P=0.052$ ). Surgical success (decline in AHI by $\geq 50 \%$ and to $\leq 20$ events/hr) also occurred more frequently in the DISE group than in the non-DISE group $(86 \%$ vs. $51 \%, P<0.001)$. However, other authors have reported that DISE made no difference in outcomes [56,57]. A conflicting report was recently published detailing the experience of 326 patients from nine centers across seven countries [68]. The investigators did not find any benefit in the DISE group (170 patients) compared to the non-DISE (156 patients) group that would support preoperative DISE. In fact, some outcome parameters favored the non-DISE group. While the strengths of this study include generalizability (It is generalizable because the samples are from multiple centers and numerous) because of its multicenter nature and rela- 
tively large sample size, its results need to be interpreted with caution. The study was not randomized. Instead patients were put into the two groups based on whether DISE had been performed, which was dependent on protocols that differed across countries and even from surgeon to surgeon. There could therefore have been selection bias, as DISE may not have been performed in patients perceived to be good surgical candidates based on other clinical parameters, and the surgical procedures performed were decided upon by individual surgeons without a common treatment algorithm. The results may reflect the standard of care and effectiveness of OSA surgery between countries, rather than truly revealing the utility—or lack thereof—of DISE.

Although DISE equips a surgeon with knowledge of obstruction patterns, an interesting study found that similar postoperative success rates and AHI could be achieved in patients with unilevel and multilevel obstruction seen on DISE after unilevel (pharyngoplasty) surgery alone. The authors suggested that multilevel surgery may not always be necessary at first, even in patients who demonstrate multilevel obstruction during DISE [69]. However, it is unknown whether the patients with multilevel obstruction would have had even better outcomes if multilevel surgery had been performed. Further multicenter prospective randomized trials with control groups who do not undergo DISE are sorely needed to investigate the true clinical impact of DISE in patients undergoing OSA surgery.

\section{ROLE OF DISE IN PLANNING UPPER AIRWAY STIMULATION}

Upper airway stimulation is a surgical technique that has emerged in recent years. Implantation of the stimulation system reduces upper airway collapsibility by stimulating the hypoglossal nerve to cause tongue protrusion and opening of the upper airway. Inspire (Inspire Medical Systems Inc., Maple Grove, MN, USA), an implantable pacemaker-like pulse-generator with sensing and stimulation leads, was the first device of its kind approved by the U.S. Food and Drug Administration in 2014 [70,71]. Mediumterm data on safety and efficacy have been promising [72]. Since then, a few similar systems have been developed and evaluated $[73,74]$. In recent individual patient-level data from four cohorts comprising 584 adults with OSA implanted with the Inspire hypoglossal nerve stimulator, treatment success (defined as a decrease in AHI by $>50 \%$ and to $<20$ events/hr) was observed in $77.1 \%$ of patients [75].

DISE was incorporated as a mandatory screening investigation in the landmark Stimulation Therapy for Apnea Reduction (STAR) trial following earlier studies that showed CCCp during DISE to be associated with poor results after upper airway stimulation $[76,77]$. One of these studies analyzed 21 CPAP-intolerant moderate to severe OSA patients who underwent DISE before im- plantation [76]. Five of the patients (23.8\%) demonstrated CCCp, of whom none achieved success or a significant change in the AHI ( $41.5 \pm 13.8$ vs. $48.1 \pm 18.7$ events/hr, $P=0.44) 6$ months post-implantation. Conversely, $81 \%$ of those without CCCp achieved success (defined as AHI $<20$ events/hr and a reduction of $\geq 50 \%$ ). As the technology undergoes further refinement and becomes more affordable, upper airway stimulation will continue to revolutionize the treatment of OSA, and the role that DISE plays in the patient selection protocol will continue to evolve.

In the event of poor treatment response after implantation despite multiple settings and titration attempts, DISE can be performed to troubleshoot the reason for failure. Palatoglossal coupling is one of the main mechanisms by which protrusion of the genioglossus increases both retrolingual and retropalatal dimensions. However, patients with persistent soft palate obstruction seen on DISE may benefit from uvulopalatopharyngoplasty with tonsillectomy [78]. Similarly, DISE can help to evaluate any residual areas of obstruction in OSA surgery non-responders [79].

\section{CONCLUSION}

High-quality clinical evidence supporting the value of DISE in guiding alternative treatments for OSA is limited. The heterogeneous and retrospective nature of many studies, as well as issues of inherent bias, has produced a bag of mixed conclusions. As a result, adoption and utilization of DISE is varied across the globe, and it is often not understood or considered relevant by many nonsurgical sleep practitioners. However, this disenchantment should be addressed because OSA is a multidisciplinary condition with complex pathophysiology and profound cardiometabolic consequences. The paradigm of OSA treatment has shifted-its objectives have gone beyond improving snoring and sleepiness alone, and now encompass bettering blood pressure control and cardiovascular outcomes. Pivotal multicenter clinical trials of CPAP therapy have thus far highlighted the high prevalence of poor adherence and the neutral effect of CPAP on cardiovascular outcomes $[80,81]$. DISE has the advantage of providing an increased understanding of a patient's upper airway mechanics via a low-risk procedure, with the possibility of using the knowledge gained to make guided prescriptions of treatment alternatives that may increase the frequency of positive outcomes. DISE has the potential to be the main driver behind the next level of care for OSA in this era of precision medicine.

\section{CONFLICT OF INTEREST}

No potential conflict of interest relevant to this article was reported. 


\section{ACKNOWLEDGMENTS}

CHL was supported by research grants from the National Medical Research Council of Singapore (Award no. NMRC/CG/C006/ 2017; NMRC/CSA-SIV/001/2018).

\section{ORCID}

Crystal SJ Cheon

https://orcid.org/0000-0002-7238-327X

Weiqiang Loke

https://orcid.org/0000-0002-1634-2932

Mark Kim ThyeThong https://orcid.org/0000-0001-7060-4270

Song TarToh

https://orcid.org/0000-0003-2077-2457

Chi-Hang Lee

https://orcid.org/0000-0001-8777-2705

\section{AUTHOR CONTRIBUTIONS}

Conceptualization; CHL, CSJC, MKTT, STT. Data curation: CSJC, CHL, WL. Funding acquisition: CHL. Writing-original draft, review, \& editing: all authors.

\section{REFERENCES}

1. Benjafield AV,Ayas NT, Eastwood PR, Heinzer R, Ip MS, Morrell MJ, et al. Estimation of the global prevalence and burden of obstructive sleep apnoea: a literature-based analysis. Lancet Respir Med. 2019 Aug;7(8):687-98.

2. BradleyTD, Floras JS. Obstructive sleep apnoea and its cardiovascular consequences. Lancet. 2009 Jan;373(9657):82-93.

3. Lee CH, Sethi R, Li R, Ho HH, Hein T, Jim MH, et al. Obstructive sleep apnea and cardiovascular events after percutaneous coronary intervention. Circulation. 2016 May;133(21):2008-17.

4. Koo CY,Aung AT, Chen Z, KristantoW, Sim HW,TamWW, et al. Sleep apnoea and cardiovascular outcomes after coronary artery bypass grafting. Heart. 2020 Oct;106(19):1495-502.

5. Rotenberg BW, Murariu D, Pang KP.Trends in CPAP adherence over twenty years of data collection: a flattened curve. J Otolaryngol Head Neck Surg. 2016 Aug;45(1):43.

6. Sutherland K, Kairaitis K,Yee BJ, Cistulli PA. From CPAP to tailored therapy for obstructive sleep Apnoea. Multidiscip Respir Med. 2018 Dec;13:44.

7. Virk JS, Kotecha B. When continuous positive airway pressure (CPAP) fails. JThorac Dis. 2016 Oct;8(10):E1112-21.

8. WatersT.Alternative interventions for obstructive sleep apnea. Cleve Clin J Med. 2019 Sep;86(9 Suppl 1):34-41.

9. Eckert DJ. Phenotypic approaches to obstructive sleep apnoea: new pathways for targeted therapy. Sleep Med Rev. 2018 Feb;37:45-59.

10. Sands SA, Edwards BA, Terrill PI, Taranto-Montemurro L, Azarbarzin A, Marques M, et al. Phenotyping pharyngeal pathophysiology using polysomnography in patients with obstructive sleep apnea. Am J Respir Crit Care Med. 2018 May;197(9):1187-97.

11. Sands SA,Terrill PI, Edwards BA,Taranto Montemurro L,Azarbarzin A, Marques M, et al. Quantifying the arousal threshold using polysomnography in obstructive sleep apnea. Sleep. 2018 Jan;41(1):zsx183.

12. Terrill PI, Edwards BA, Nemati S, Butler JP, Owens RL, Eckert DJ, et al. Quantifying the ventilatory control contribution to sleep ap- noea using polysomnography. Eur Respir J. 2015 Feb;45(2):408-18.

13. Genta PR, Sands SA, Butler JP, Loring SH, Katz ES, Demko BG, et al. Airflow shape is associated with the pharyngeal structure causing OSA. Chest. 2017 Sep;152(3):537-46.

14. Vroegop AV,Vanderveken OM, Boudewyns AN, Scholman J, Saldien V,Wouters K, et al. Drug-induced sleep endoscopy in sleep-disordered breathing: report on 1,249 cases. Laryngoscope. 2014 Mar;124(3): 797-802.

15. Woodson BT, Wooten MR.A multisensor solid-state pressure manometer to identify the level of collapse in obstructive sleep apnea. Otolaryngol Head Neck Surg. 1992 Nov;107(5):651-6.

16. De Vito A, Carrasco Llatas M, Ravesloot MJ, Kotecha B, De Vries N, Hamans E, et al. European position paper on drug-induced sleep endoscopy: 2017 update. Clin Otolaryngol. 2018 Dec;43(6):1541-52.

17. DeVito A, Carrasco Llatas M,Vanni A, Bosi M, Braghiroli A, Campanini A, et al. European position paper on drug-induced sedation endoscopy (DISE). Sleep Breath. 2014 Sep;18(3):453-65.

18. Kotecha B, DeVito A. Drug induced sleep endoscopy: its role in evaluation of the upper airway obstruction and patient selection for surgical and non-surgical treatment. JThorac Dis. 2018 Jan;10(Suppl 1): S40-7.

19. Blumen M, Bequignon E, Chabolle F. Drug-induced sleep endoscopy: a new gold standard for evaluating OSAS? Part II: results. Eur Ann Otorhinolaryngol Head Neck Dis. 2017 Apr;134(2):109-15.

20. Croft CB, Pringle M. Sleep nasendoscopy: a technique of assessment in snoring and obstructive sleep apnoea. Clin Otolaryngol Allied Sci. 1991 Oct;16(5):504-9.

21. Amos JM, Durr ML, Nardone HC, Baldassari CM, Duggins A, Ishman SL. Systematic review of drug-induced sleep endoscopy scoring systems. Otolaryngol Head Neck Surg. 2018 Feb;158(2):240-8.

22. Dijemeni E, D’Amone G, Gbati I. Drug-induced sedation endoscopy (DISE) classification systems: a systematic review and meta-analysis. Sleep Breath. 2017 Dec;21(4):983-94.

23. Lee $\mathrm{CH}$, Seay EG, Dedhia RC. IMAGES: drug-induced sleep endoscopy. An investigative tool for mechanisms of PAP failure. J Clin Sleep Med. 2019 Jan;15(1):171-2.

24. Ramar K, Dort LC, Katz SG, Lettieri CJ, Harrod CG, Thomas SM, et al. Clinical practice guideline for the treatment of obstructive sleep apnea and snoring with oral appliance therapy: an update for 2015. J Clin Sleep Med. 2015 Jul;11(7):773-827.

25. Brown EC, Cheng S, McKenzie DK, Butler JE, Gandevia SC, Bilston LE. Tongue and lateral upper airway movement with mandibular advancement. Sleep. 2013 Mar;36(3):397-404.

26. Chan AS, Sutherland K, Schwab RJ, Zeng B, Petocz P, Lee RW, et al. The effect of mandibular advancement on upper airway structure in obstructive sleep apnoea. Thorax. 2010 Aug;65(8):726-32.

27. Sutherland K, Deane SA, Chan AS, Schwab RJ, Ng AT, Darendeliler MA, et al. Comparative effects of two oral appliances on upper airway structure in obstructive sleep apnea. Sleep. 2011 Apr;34(4):46977.

28. Phillips CL, Grunstein RR, Darendeliler MA, Mihailidou AS, Srinivasan VK, Yee BJ, et al. Health outcomes of continuous positive airway pressure versus oral appliance treatment for obstructive sleep apnea: a randomized controlled trial. Am J Respir Crit Care Med. 2013 Apr; 187(8):879-87.

29. Johal A, Battagel JM, Kotecha BT. Sleep nasendoscopy: a diagnostic tool for predicting treatment success with mandibular advancement splints in obstructive sleep apnoea. Eur J Orthod. 2005 Dec;27(6): 607-14.

30. Huntley C, Cooper J, Stiles M, Grewal R, Boon M. Predicting success of oral appliance therapy in treating obstructive sleep apnea using drug-induced sleep endoscopy. J Clin Sleep Med. 2018 Aug;14(8): 1333-7.

31. Vroegop AV, Vanderveken OM, Dieltjens M, Wouters K, Saldien V, 
Braem MJ, et al. Sleep endoscopy with simulation bite for prediction of oral appliance treatment outcome. J Sleep Res. 2013 Jun;22(3): 348-55.

32. Vonk PE, Uniken Venema JA, Hoekema A, Ravesloot MJ, van de Velde-Muusers JA, de Vries N. Jaw thrust versus the use of a boil-andbite mandibular advancement device as a screening tool during druginduced sleep endoscopy. J Clin Sleep Med. 2020 Jul;16(7):1021-7.

33. Vroegop AV, Vanderveken OM,Verbraecken JA. Drug-induced sleep endoscopy: evaluation of a selection tool for treatment modalities for obstructive sleep apnea. Respiration. 2020;99(5):451-7.

34. Park D, Kim JS, Heo SJ.The effect of the modified jaw-thrust maneuver on the depth of sedation during drug-induced sleep endoscopy. J Clin Sleep Med. 2019 Oct;15(10):1503-8.

35. Op de Beeck S, Dieltjens M,Verbruggen AE,Vroegop AV, Wouters K, Hamans E, et al. Phenotypic labelling using drug-induced sleep endoscopy improves patient selection for mandibular advancement device outcome: a prospective study. J Clin Sleep Med. 2019 Aug; 15(8):1089-99.

36. Cartwright RD. Effect of sleep position on sleep apnea severity. Sleep. 1984;7(2):110-4.

37. Mador MJ, KufelTJ, Magalang UJ, Rajesh SK, WatweV, Grant BJ. Prevalence of positional sleep apnea in patients undergoing polysomnography. Chest. 2005 Oct;128(4):2130-7.

38. RichardW, Kox D, den Herder C, Laman M, vanTinteren H, deVries $\mathrm{N}$. The role of sleep position in obstructive sleep apnea syndrome. Eur Arch Otorhinolaryngol. 2006 Oct;263(10):946-50.

39. Eijsvogel MM, Ubbink R, Dekker J, Oppersma E, de Jongh FH, van der Palen J, et al. Sleep position trainer versus tennis ball technique in positional obstructive sleep apnea syndrome. J Clin Sleep Med. 2015 Jan;11(2):139-47.

40. Vonk PE, van de Beek MJ, Ravesloot MJ, de Vries N. Drug-induced sleep endoscopy: new insights in lateral head rotation compared to lateral head and trunk rotation in (non)positional obstructive sleep apnea patients. Laryngoscope. 2019 Oct;129(10):2430-5.

41. Safiruddin F, Koutsourelakis I, de Vries N. Analysis of the influence of head rotation during drug-induced sleep endoscopy in obstructive sleep apnea. Laryngoscope. 2014 Sep;124(9):2195-9.

42. Victores AJ, Hamblin J, Gilbert J, Switzer C,Takashima M. Usefulness of sleep endoscopy in predicting positional obstructive sleep apnea. Otolaryngol Head Neck Surg. 2014 Mar;150(3):487-93.

43. Ravesloot MJ, de Vries N. One hundred consecutive patients undergoing drug-induced sleep endoscopy: results and evaluation. Laryngoscope. 2011 Dec;121(12):2710-6.

44. Dieltjens M, Vroegop AV, Verbruggen AE, Wouters K, Willemen M, De Backer WA, et al. A promising concept of combination therapy for positional obstructive sleep apnea. Sleep Breath. 2015 May;19(2): 637-44.

45. Kotecha BT, Hall AC. Role of surgery in adult obstructive sleep apnoea. Sleep Med Rev. 2014 Oct;18(5):405-13.

46. Soares D, Folbe AJ, Yoo G, Badr MS, Rowley JA, Lin HS. Drug-induced sleep endoscopy vs awake Müller's maneuver in the diagnosis of severe upper airway obstruction. Otolaryngol Head Neck Surg. 2013 Jan;148(1):151-6.

47. Woodson BT, Wooten MR. Comparison of upper-airway evaluations during wakefulness and sleep. Laryngoscope. 1994 Jul;104(7):821-8.

48. Liu SY, Huon LK, IwasakiT, Yoon A, Riley R, Powell N, et al. Efficacy of maxillomandibular advancement examined with drug-induced sleep endoscopy and computational fluid dynamics airflow modeling. Otolaryngol Head Neck Surg. 2016 Jan;154(1):189-95.

49. Liu SY, Huon LK, Powell NB, Riley R, Cho HG, Torre C, et al. Lateral pharyngeal wall tension after maxillomandibular advancement for obstructive sleep apnea is a marker for surgical success: observations from drug-induced sleep endoscopy. J Oral Maxillofac Surg. 2015 Aug;73(8):1575-82.
50. Aktas O, Erdur O, Cirik AA, Kayhan FT. The role of drug-induced sleep endoscopy in surgical planning for obstructive sleep apnea syndrome. Eur Arch Otorhinolaryngol. 2015 Aug;272(8):2039-43.

51. Meraj TS, Muenz DG, Glazer TA, Harvey RS, Spector ME, Hoff PT. Does drug-induced sleep endoscopy predict surgical success in transoral robotic multilevel surgery in obstructive sleep apnea? Laryngoscope. 2017 Apr;127(4):971-6.

52. Ha JG, LeeY, Nam JS, Park JJ, Yoon JH, Kim CH, et al. Can drug-induced sleep endoscopy improve the success rates of tongue base surgery? J Otolaryngol Head Neck Surg. 2020 Feb;49(1):8.

53. Green KK, Kent DT, D’Agostino MA, Hoff PT, Lin HS, Soose RJ, et al. Drug-induced sleep endoscopy and surgical outcomes: a multicenter cohort study. Laryngoscope. 2019 Mar;129(3):761-70.

54. Eichler C, Sommer JU, Stuck BA, Hormann K, Maurer JT. Does druginduced sleep endoscopy change the treatment concept of patients with snoring and obstructive sleep apnea? Sleep Breath. 2013 Mar; 17(1):63-8.

55. Gillespie MB, Reddy RP,White DR, Discolo CM, Overdyk FJ, Nguyen SA. A trial of drug-induced sleep endoscopy in the surgical management of sleep-disordered breathing. Laryngoscope. 2013 Jan;123(1): 277-82.

56. Yilmaz YF, Kum RO, Ozcan M, GungorV, UnalA. Drug-induced sleep endoscopy versus Muller maneuver in patients with retropalatal obstruction. Laryngoscope. 2015 Sep;125(9):2220-5.

57. CertalVF, Pratas R, Guimaraes L, Lugo R, Tsou Y, Camacho M, et al. Awake examination versus DISE for surgical decision making in patients with OSA: a systematic review. Laryngoscope. 2016 Mar;126(3): 768-74.

58. Gazzaz MJ, Isaac A, Anderson S, Alsufyani N, AlrajhiY, El-Hakim H. Does drug-induced sleep endoscopy change the surgical decision in surgically naive non-syndromic children with snoring/sleep disordered breathing from the standard adenotonsillectomy? A retrospective cohort study. J Otolaryngol Head Neck Surg. 2017 Feb;46(1):12.

59. Galluzzi F, Pignataro L, Gaini RM, Garavello W. Drug induced sleep endoscopy in the decision-making process of children with obstructive sleep apnea. Sleep Med. 2015 Mar;16(3):331-5.

60. Wilcox LJ, Bergeron M, Reghunathan S, Ishman SL. An updated review of pediatric drug-induced sleep endoscopy. Laryngoscope Investig Otolaryngol. 2017 Nov;2(6):423-31.

61. Kirkham E, Ma CC, Filipek N, Horn DL, Johnson K, Chen ML, et al. Polysomnography outcomes of sleep endoscopy-directed intervention in surgically naïve children at risk for persistent obstructive sleep apnea. Sleep Breath. 2020 Sep;24(3):1143-50.

62. Dmowska J, Larson SR, Gillespie MB, Sheyn A. Effect of drug induced sleep endoscopy on intraoperative decision making in pediatric sleep surgery. Int J Pediatr Otorhinolaryngol. 2020 Mar;130:109810.

63. Chan DK, Truong MT, Koltai PJ. Supraglottoplasty for occult laryngomalacia to improve obstructive sleep apnea syndrome. Arch Otolaryngol Head Neck Surg. 2012 Jan;138(1):50-4.

64. Revell SM, ClarkWD. Late-onset laryngomalacia: a cause of pediatric obstructive sleep apnea. Int J Pediatr Otorhinolaryngol. 2011 Feb; 75(2):231-8.

65. Richter GT, Rutter MJ, deAlarcon A, Orvidas LJ, Thompson DM. Late-onset laryngomalacia: a variant of disease. Arch Otolaryngol Head Neck Surg. 2008 Jan;134(1):75-80.

66. Hessel NS, de Vries N. Results of uvulopalatopharyngoplasty after diagnostic workup with polysomnography and sleep endoscopy: a report of 136 snoring patients. Eur Arch Otorhinolaryngol. 2003 Feb; 260(2):91-5.

67. Huntley C, Chou D, Doghramji K, Boon M. Preoperative drug induced sleep endoscopy improves the surgical approach to treatment of obstructive sleep apnea. Ann Otol Rhinol Laryngol. 2017 Jun;126(6): 478-82.

68. Pang KP, Baptista PM, Olszewska E, Braverman I, Carrasco-Llatas M, 
Kishore S, et al. Does drug-induced sleep endoscopy affect surgical outcome? A multicenter study of 326 obstructive sleep apnea patients. Laryngoscope. 2020 Feb;130(2):551-5.

69. Hsu YS, Jacobowitz O. Does sleep endoscopy staging pattern correlate with outcome of advanced palatopharyngoplasty for moderate to severe obstructive sleep apnea? J Clin Sleep Med. 2017 Oct;13(10): 1137-44.

70. Strollo PJ Jr, Soose RJ, Maurer JT, de Vries N, Cornelius J, Froymovich $\mathrm{O}$, et al. Upper-airway stimulation for obstructive sleep apnea. $\mathrm{N}$ Engl J Med. 2014 Jan;370(2):139-49.

71. Wray CM,Thaler ER. Hypoglossal nerve stimulation for obstructive sleep apnea: a review of the literature. World J Otorhinolaryngol Head Neck Surg. 2016 Dec;2(4):230-3.

72. Steffen A, Sommer JU, Hofauer B, Maurer JT, Hasselbacher K, Heiser C. Outcome after one year of upper airway stimulation for obstructive sleep apnea in a multicenter German post-market study. Laryngoscope. 2018 Feb;128(2):509-15.

73. Sommer JU, Hormann K. Innovative surgery for obstructive sleep apnea: nerve stimulator. Adv Otorhinolaryngol. 2017;80:116-24.

74. Eastwood PR, Barnes M, MacKay SG, Wheatley JR, Hillman DR, Nguyen XL, et al. Bilateral hypoglossal nerve stimulation for treatment of adult obstructive sleep apnoea. Eur Respir J. 2020 Jan;55(1): 1901320.

75. Kent DT, Carden KA, Wang L, Lindsell CJ, Ishman SL. Evaluation of hypoglossal nerve stimulation treatment in obstructive sleep apnea.
JAMA Otolaryngol Head Neck Surg. 2019 Sep;145(11):1044-52.

76. Vanderveken OM, Maurer JT, Hohenhorst W, Hamans E, Lin HS, Vroegop AV, et al. Evaluation of drug-induced sleep endoscopy as a patient selection tool for implanted upper airway stimulation for obstructive sleep apnea. J Clin Sleep Med. 2013 May;9(5):433-8.

77. Van de Heyning PH, Badr MS, Baskin JZ, Cramer Bornemann MA, De Backer WA, Dotan Y, et al. Implanted upper airway stimulation device for obstructive sleep apnea. Laryngoscope. 2012 Jul;122(7): 1626-33.

78. Steffen A, Abrams N, Suurna MV, Wollenberg B, Hasselbacher K. Upper-airway stimulation before, after, or without uvulopalatopharyngoplasty: a two-year perspective. Laryngoscope. 2019 Feb;129(2): 514-8.

79. Kezirian EJ. Nonresponders to pharyngeal surgery for obstructive sleep apnea: insights from drug-induced sleep endoscopy. Laryngoscope. 2011 Jun;121(6):1320-6.

80. McEvoy RD, Antic NA, Heeley E, LuoY, Ou Q, Zhang X, et al. CPAP for prevention of cardiovascular events in obstructive sleep apnea. $\mathrm{N}$ Engl J Med. 2016 Sep;375(10):919-31.

81. Sanchez-de-la-Torre M, Sanchez-de-la-Torre A, Bertran S, Abad J, Duran-Cantolla J, CabriadaV, et al. Effect of obstructive sleep apnoea and its treatment with continuous positive airway pressure on the prevalence of cardiovascular events in patients with acute coronary syndrome (ISAACC study): a randomised controlled trial. Lancet Respir Med. 2020 Apr;8(4):359-67. 